\title{
Non-ciliary Roles of IFT Proteins in Cell Division and Polycystic Kidney Diseases
}

\author{
Benjamin Vitre*, Audrey Guesdon and Benedicte Delaval* \\ CRBM, Univ Montpellier, CNRS, Montpellier, France
}

Cilia are small organelles present at the surface of most differentiated cells where they act as sensors for mechanical or biochemical stimuli. Cilia assembly and function require the Intraflagellar Transport (IFT) machinery, an intracellular transport system that functions in association with microtubules and motors. If IFT proteins have long been studied for their ciliary roles, recent evidences indicate that their functions are not restricted to the cilium. Indeed, IFT proteins are found outside the ciliary compartment where they are involved in a variety of cellular processes in association with nonciliary motors. Recent works also provide evidence that non-ciliary roles of IFT proteins

\section{OPEN ACCESS}

Edited by:

Rytis Prekeris,

University of Colorado Denver,

United States

Reviewed by:

Chad G. Pearson,

University of Colorado, United States Brian Paul Ceresa,

University of Louisville, United States

*Correspondence: Benjamin Vitre

benjamin.vitre@crbm.cnrs.fr Benedicte Delaval

benedicte.delaval@crbm.cnrs.fr

Specialty section:

This article was submitted to

Membrane Traffic,

a section of the journal

Frontiers in Cell and Developmental

Biology

Received: 30 June 2020

Accepted: 26 August 2020

Published: 22 September 2020

Citation:

Vitre B, Guesdon A and Delaval B

(2020) Non-ciliary Roles of IFT

Proteins in Cell Division

and Polycystic Kidney Diseases.

Front. Cell Dev. Biol. 8:578239.

doi: 10.3389/fcell.2020.578239 could be responsible for the development of ciliopathies related phenotypes including polycystic kidney diseases. In this review, we will discuss the interactions of IFT proteins with microtubules and motors as well as newly identified non-ciliary functions of IFT proteins, focusing on their roles in cell division. We will also discuss the potential contribution of non-ciliary IFT proteins functions to the etiology of kidney diseases.

Keywords: microtubule - associated proteins, cell division, ciliopathies, molecular motor, intraflagellar transport

\section{INTRODUCTION}

Scientists have identified and described flagella since hundreds of years. However, the development of novel staining methods and the advance of microscopy techniques, in the second half of the 19th century allowed rapid progress in the description of this organelle. Importantly, those technological advances elicited the distinction between cilia and flagella and linked them to basal bodies and centrosomes (Bloodgood, 2009). In the second half of the 20th century, the use of transmission electron microscopy in cell biology revealed the presence of cilia in a variety of tissues and organisms. They organize in $9+2$ and $9+0$ tubular "fibrils," the axonemes, emanating from basal bodies and extending longitudinally into cilia and flagella (Manton and Clarke, 1952; Fawcett and Porter, 1954; Barnes, 1961; Sorokin, 1962; Bloodgood, 2009). Those "fibrils" were later termed microtubules (Ledbetter and Porter, 1963; Slautterback, 1963). In the 1990's and 2000's, multiple studies linking cilia and cilia proteins to development and disease established the primary cilium as a central player in cell and tissue homeostasis.

First, the discovery of an intraflagellar transport (IFT) system within the flagellum of Chlamydomonas reinhardtii (Kozminski et al., 1993), its importance for cilia and flagella assembly (Cole et al., 1998; Pazour et al., 1998, 2000; Piperno et al., 1998) and the link between IFT proteins and ciliopathies attracted a great amount of attention on cilia (Pazour et al., 2000; Reiter and Leroux, 2017). IFT depends on three motors, homodimeric kinesin 2, heterotrimeric kinesin 2 and dynein 2 that move along axoneme microtubules. These motors carry IFT trains containing IFT proteins (Prevo et al., 2017). While the exact functions of the proteins involved 
in IFT are still being precisely studied, it is clearly established that they are essential for cilia formation and function (Reiter and Leroux, 2017). Second, the discovery that cilia can act as flow sensors (Praetorius and Spring, 2001, 2003) having a major impact on mammalian development asserted cilia as a central organelle for development (McGrath et al., 2003; Yoshiba et al., 2012; Ferreira et al., 2019). Third, the discovery that the IFT machinery and the primary cilium were involved in Sonic Hedgehog signaling, an essential developmental pathway associated with ciliopathy related phenotypes (Varjosalo and Taipale, 2008) and frequently upregulated in cancer, further raised attention on this organelle (Huangfu et al., 2003; Corbit et al., 2005). Later works also linked additional signaling pathways, such as wnt or notch, to cilia (Liu et al., 2018).

Driven by the identification of those three essential aspects, many studies have since been performed in order to understand cilia biogenesis and functions and their link to diseases. However, it also appears from this abundant literature that, when it comes to genes involved in ciliopathies, there is no simple relationship between mutations within a gene and the associated phenotypes (Reiter and Leroux, 2017). For example, mutations in IFT172 can result in a variety of phenotypes including retinal degeneration (retinis pigmentosa), obesity and polydactyly (Bardet Biedl Syndrome) (Bujakowska et al., 2015) or skeletal abnormalities, nephronophthisis and other clinical features associated with Jeune syndrome or Meinzer-Saldino syndrome (Halbritter et al., 2013). To explain this disconnection between mutations and phenotypes, it was proposed that stochastic events during the development of an individual harboring the mutation or that the genetic background of the individual could result in various phenotypes (Ben-Omran et al., 2015; Ramsbottom et al., 2015, 2020). As an alternative, it was also proposed that mutations in ciliary proteins could impair non-ciliary functions that may contribute to ciliopathies related phenotypes (Vertii et al., 2015; Hua and Ferland, 2018). Indeed, there is now increasing evidence that IFT proteins also function outside the cilium. IFT proteins are, for example, involved in the regulation of microtubule dynamics and/or organization in interphase cells (Bizet et al., 2015; Boehlke et al., 2015; Dupont et al., 2019). They are also required for specialized cellular functions such as the formation of the immune synapse in T cells (Finetti et al., 2009, 2014), the inflammatory response (McFie et al., 2020), the regulation of cell cycle progression (Robert et al., 2007) or cell division (Jonassen et al., 2008; Delaval et al., 2011; Borovina and Ciruna, 2013; Taulet et al., 2017, 2019; Vitre et al., 2020). We will summarize here our current knowledge on how IFT proteins interact and regulate microtubules and motors. We will review the current literature on their non-ciliary functions focusing on their roles in cell division. We will finally discuss how perturbations of the non-ciliary functions of IFT proteins could be relevant to cystic kidney diseases.

\section{COMPOSITION OF IFT TRAINS IN CILIA}

Cilia and flagella are evolutionarily conserved microtubule-based organelles found on eukaryotic cells. Since axonemal elongation occurs at the distal plus end of microtubules, ciliary assembly requires active transport of proteins from the base to the tip of the cilium along the axoneme. IFT corresponds to the continuous and bi-directional movement of large macromolecular assemblies under the ciliary membrane required for protein trafficking inside cilia (Iomini et al., 2001; Pedersen et al., 2005; Engel et al., 2009; Pigino et al., 2009; Buisson et al., 2013). Those particles, known as IFT trains, were first identified in the flagellum of Chlamydomonas reinhardtii by Differential Interference Contrast (DIC) microscopy (Kozminski et al., 1993). In this context, they were shown to mediate interactions between motors and cargoes that need to be delivered to the ciliary tip. Later studies showed that anterograde transport from the base to the ciliary tip is based on kinesin-2 motors (Walther et al., 1994; Kozminski et al., 1995; Cole et al., 1998), while retrograde transport toward the ciliary base is dependent on dynein-2 motors (Pazour et al., 1998, 1999; Perrone et al., 2003; Hou et al., 2004). Biochemical, structural and functional analysis of those complexes revealed several differences between anterograde and retrograde IFT complexes. Indeed, distinction was made between long and narrow IFT-A trains responsible for retrograde transport, and short and compact IFT-B trains responsible for anterograde transport (Cole et al., 1998; Stepanek and Pigino, 2016). IFT-A and IFT-B complexes differ in their molecular composition (Figure 1; Taschner and Lorentzen, 2016). Despite those differences, IFT proteins are highly conserved across species. Several protein-protein interaction motifs were identified as tetratricopeptide repeats, $\mathrm{WD}-40$ repeats and coiled-coils that ensure the formation of IFT complexes and the interactions of IFT complexes with many cargoes and motors (Figure 1; Jékely and Arendt, 2006; Taschner et al., 2012).

\section{CILIARY AND NON-CILIARY INTERACTIONS OF IFT PROTEINS WITH TUBULIN AND MICROTUBULES}

Tubulin was shown to be an IFT cargo in Chlamydomonas reinhardtii (Craft et al., 2015) and C. elegans (Hao et al., 2011). Indeed, it is the most abundant ciliary protein that needs to be actively transported from the base to the tip of the cilium to ensure ciliary elongation. The interaction between soluble $\alpha \beta$-tubulin or polymerized microtubules and the IFT machinery is mediated by a highly conserved tubulinbinding module constituted by functional domains of IFT81 and IFT74 proteins. The first part of the module contains the $\mathrm{N}$-terminal tubulin-binding calponin homology domain of IFT81 that recognizes $\alpha \beta$-tubulin dimers. The second part contains the highly basic N-terminal domain of IFT74 that interacts with the E-hook of $\beta$-tubulin and therefore increases the binding affinity of the module to tubulin dimers (Bhogaraju et al., 2013; Kubo et al., 2016; Weghe et al., 2020). However, the IFT74IFT81 module has no ortholog in Drosophila melanogaster (van Dam et al., 2013; Bhogaraju et al., 2014). This suggests that other microtubule/tubulin binding sites might exist within IFT complexes. Indeed, IFT54/DYF-11/MIP-T3 was also proposed to be a binding partner of tubulin and actin (Guo et al., 2010). The binding activity with microtubule/tubulin was verified (Ling and Goeddel, 2000) and localized in the N-terminal 


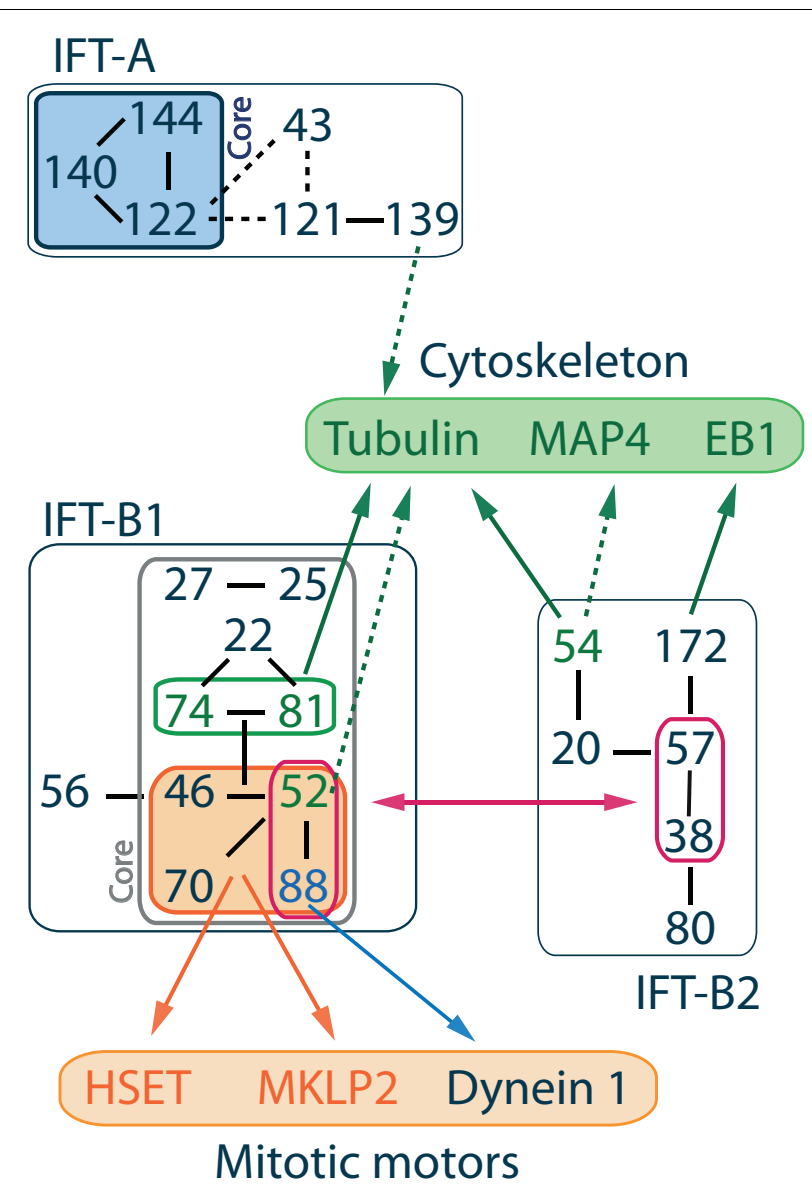

FIGURE 1 | IFT complex overall organization and interactions with cytoskeleton components and mitotic motors. IFT proteins are divided into 2 classes. IFT-A complex is made of 6 subunits IFT144-140-139-122-121-43 where IFT144-140-122 form the core complex (Mukhopadhyay et al., 2010; Behal et al., 2012) and IFT139-120-43 form a peripheral subcomplex (Hirano et al., 2017). IFT-B complex is made of 16 subunits, and its overall architecture determined by VIP (visible immunoprecipitation) assays (Katoh et al., 2016), X-ray (Taschner et al., 2016), and interactome analysis (Boldt et al., 2016) reveals the presence of two stable subcomplexes. IFT-B1 is salt stable, its core complex is made of 9 subunits IFT88-81-74-70-52-46-27-25-22 (Lucker et al., 2005; Wang et al., 2009; Bhogaraju et al., 2011; Taschner et al., 2014; Wachter et al., 2019), while IFT-B2 is the peripheral complex made of 6 subunits IFT172-80-57-54-38-20 (Baker et al., 2003; Krock and Perkins, 2008; Taschner et al., 2016, 2018). The IFT-B2 central dimer IFT57-38 in association with the N-terminal domain of IFT52 and IFT88 mediates the interaction between both IFT-B subcomplexes (Taschner et al., 2016). Within IFT-B1, a stable tetrameric complex made of IFT88-70-52-46 was identified (Lucker et al., 2010; Taschner et al., 2011, 2014). Interactions between IFT machinery and mitotic motors are mediated by this tetramer (Delaval et al., 2011; Taulet et al., 2017; Vitre et al., 2020). Interactions with cytoskeleton components are mainly mediated by the IFT-B core complex, although the member of the IFT-A complex IFT139 is proposed to be a microtubule/tubulin binding partner (see text for details). Solid lines between IFT proteins indicate validated interactions, while dashed lines indicate putative interactions.

part of IFT54/DYF-11/MIP-T3 (Taschner et al., 2016). This additional binding site could be responsible for the binding of two tubulin molecules per IFT-B complex. However, since IFT54 is not necessary for tubulin trafficking during ciliogenesis
(Zhu et al., 2017), other microtubule/tubulin binding partners were proposed. IFT139 is for example able to pull down several ciliary precursors including tubulin (Qin et al., 2004). IFT57 and IFT38/DYF-3 also present a divergent calponin homology domain identified in several tubulin binding proteins (Schou et al., 2014; Taschner et al., 2016). However, the tubulin binding capacity of IFT57 and IFT38 have yet to be tested experimentally.

Through those interactions, IFT proteins can play regulatory roles on microtubules. As shown in Chlamydomonas reinhardtii, IFT proteins function as a tubulin transporter. They regulate microtubule polymerization and thus cilia growth, for example by concentrating soluble tubulin in cilia (Craft et al., 2015). Different studies also highlight the role of individual IFT proteins on the microtubule cytoskeleton. IFT70 is, for example, a regulator of ciliary tubulin polyglutamylation that is necessary for proper ciliogenesis in vertebrates (Pathak et al., 2007). IFT54 plays a role in the regulation of cytoplasmic microtubule dynamics by modulating the activity of MAP4, a microtubule-associated protein (MAP) stabilizing cytoplasmic microtubules (Bizet et al., 2015). IFT172 also interacts with the microtubule plus-end binding protein EB1 known to modulate microtubule dynamics and that localizes at the flagella tip (Pedersen et al., 2003, 2005). Altogether, this literature indicates that IFT proteins can play regulatory roles on the microtubule cytoskeleton both inside and outside the cilium by interacting with tubulin and microtubules directly or through MAPs.

\section{NON-CILIARY FUNCTIONS OF IFT PROTEINS IN DIVIDING CELLS}

While most of the studies regarding IFT protein characterization were done in the context of cilia, it rapidly appeared that they could also function in non-ciliated dividing cells. Indeed, IFT52 localizes at the centrosomes constituting the mitotic spindle poles in Chlamydomonas reinhardtii (Deane et al., 2001). IFT74 localizes at the centrosome of mitotic human endothelial HUVEC cells (Iomini et al., 2004). IFT20 localizes at the Golgi and at the centrosome of mitotic RPE-1 cells (Follit et al., 2006). Finally, IFT88 also localizes at the centrosome of dividing RPE-1 and LLC-PK1 cells (Robert et al., 2007; Delaval et al., 2011).

In agreement with these localizations, IFT proteins were shown to contribute to various processes in dividing cells in association with microtubules and motors (Figure 2). Indeed, several works showed that IFT proteins contribute to proper mitotic spindle geometry and dynamics. IFT88 was initially described for its role in mitotic spindle orientation in kidney cells and zebrafish embryos (Delaval et al., 2011). In this context, IFT88, in association with the non-ciliary cytoplasmic dynein 1 , is required for the re-localization of peripheral microtubule clusters to the poles of the mitotic spindle. This ensures efficient enrichment of astral microtubules and proper spindle orientation (Delaval et al., 2011). More recently, IFT88 was shown to control efficient mitotic spindle dynamics in prometaphase (Taulet et al., 2019). More specifically, IFT88 is required at minus end of kinetochore-bound-microtubules to recruit the microtubule binding protein NuMA (Nuclear mitotic apparatus protein) and 
cytoplasmic dynein 1. This recruitment ensures kinetochore fibers organization into spindle poles and proper chromosomes alignment (Hueschen et al., 2017). Finally, the IFT88-70-52-46 tetramer was shown to be necessary for efficient clustering of centrosomes during mitosis in cells harboring supernumerary centrosomes (Vitre et al., 2020). More specifically, the IFT proteins tetramer interacts directly with the mitotic motor HSET to ensure efficient centrosome clustering in mitosis. This mechanism is essential to ensure the proliferation and survival of cancer cells harboring supernumerary centrosomes. Of note, as opposed to this pro-survival role of IFT-B proteins in cancer cells with extra centrosomes, multiple studies have also shown that down-regulation of IFT88 drives pro-invasive phenotypes and cell proliferation. This was shown in hepatocellular carcinomas (Isfort et al., 1997; Huang Q. et al., 2017; Huang Y. et al., 2017; You et al., 2017) and in Hela cells (Robert et al., 2007).

Interestingly, several works also indicated that IFT proteins could function at later stages of mitosis to control cytokinesis. Indeed, several IFT proteins concentrate at the cleavage furrow in Chlamydomonas reinhardtii (Wood et al., 2012) and IFT88 was shown to control symmetrical cleavage furrow ingression during cytokinesis in mammalian kidney cells (Taulet et al., 2017). Mechanistically, the IFT88-70-52-46 complex directly interacts with the motor KIF20A/MKLP2 (Mitotic Kinesin-Like Protein 2 ). This is required for the timely and uniform relocalization of the Aurora B kinase toward the central spindle after anaphase onset, a mechanism that initiates cytokinetic furrow ingression. While IFT88 depletion does not prevent cytokinesis per se, this study showed that IFT proteins were required to control cell division geometry by controlling cleavage furrow ingression. It further showed that perturbations of cytokinesis geometry could have important detrimental consequences on $3 \mathrm{D}$ tissue organization.

Altogether those results unraveled key roles for IFT proteins in controlling cell division geometry in association with microtubules and motors.

\section{A ROLE FOR IFT PROTEINS IN CILIARY AND NON-CILIARY MOTORS REGULATION?}

Intraflagellar transport proteins have long been described as adapters between cargoes and motors. In cilia, proper movement of IFT complexes along the ciliary axoneme is mediated by interactions between IFT trains and molecular motors. Dynein2 is responsible for the retrograde movement of IFT trains from the tip to the base of the cilia and needs to be transported to the ciliary tip as an inactive cargo via the anterograde IFT. This anterograde transport is mediated by kinesin motors. Kinesin2 motors have different binding partners within the IFT-B complex. The kinesin-2 trimer KIF3A/KIF3B/KIFAP3 was shown to interact with the IFT88-52-57-38 tetramer (Funabashi et al., 2018). The coiled coil domain of IFT20 was identified as bridging KIF3B to IFT particles via its interaction with IFT57 (Baker et al., 2003; Krock and Perkins, 2008). The mammalian homodimeric kinesin-2 KIF17 was shown to directly interact with the IFT4656 dimer and proposed to act as a cargo rather than a motor of IFT-B. This interaction is essential for its entry in the ciliary compartment (Howard et al., 2013; Funabashi et al., 2017).

However, the hypothesis that IFT subunits could directly impact and regulate the activity of ciliary motors was also proposed (Imanishi et al., 2006). In C. elegans, the IFT kinesin OSM3 exists in an autoinhibited form, and the binding of an IFT cargo, IFT70/DYF1 would relieve the autoinhibition of the motor. This hypothesis was recently confirmed in vitro (Mohamed et al., 2018). This work showed that OSM3 directly interacts with IFT70, leading to its activation. More specifically, IFT70 was shown to mediate the recruitment of the IFT-B subcomplex IFT88-70-52-46 to OSM3, triggering the full activation of the motor. This study demonstrated for the first time a direct regulatory role for IFT proteins on the activity of a ciliary kinesin.

Importantly, this work now raises the question of the potential impact of IFT proteins on the regulation and/or activation of other molecular motors. These include the recently characterized mitotic motors interacting with IFT proteins. However, whether IFT proteins could function as trafficking modules regulating motor activity and subsequently facilitating ciliary and nonciliary transport will have to be addressed.

\section{NON-CILIARY ROLES OF IFT PROTEINS IN CYSTIC KIDNEY DISEASES}

The various functions of IFT proteins outside cilia raised the question of their contribution to tissue homeostasis and diseases (Figure 2).

First clues in favor of a contribution of cell division defects induced by IFT proteins perturbations to kidney tissue disorganization came from the observation of abnormal cell division orientation in animal models of IFT depletion. Defects in spindle orientation were observed in IFT20 knock-out mice developing kidney cysts, although in this case the exact molecular mechanisms responsible for the spindle misorientation was not elucidated (Jonassen et al., 2008). Similarly, defects in mitotic spindle orientation were observed in the pronephric ducts of IFT88 depleted zebrafish embryos (Delaval et al., 2011). This suggested that IFT perturbations could contribute to kidney cyst initiation by controlling proper cell division orientation. The role of IFT88 in mitotic spindle orientation independently of ciliary defects was later confirmed in vivo in zebrafish embryos (Borovina and Ciruna, 2013). Of note, polycystic kidney diseases in animal models were also proposed to result from planar cell polarity (PCP) defects leading to abnormal cell division orientations (Fischer et al., 2006). Along this line, some genes involved in cystic kidney diseases appear to have no apparent function in ciliogenesis but to be necessary for cell division orientation and/or PCP pathways signaling. For example seahorse, a zebrafish cystic kidney gene, while being linked to cilia dependent Wnt signaling and PCP perturbation, does not appear to be involved in ciliary assembly (Kishimoto et al., 2008). Importantly, defects in spindle orientation following IFT depletion is not observed in all models of IFT perturbations 


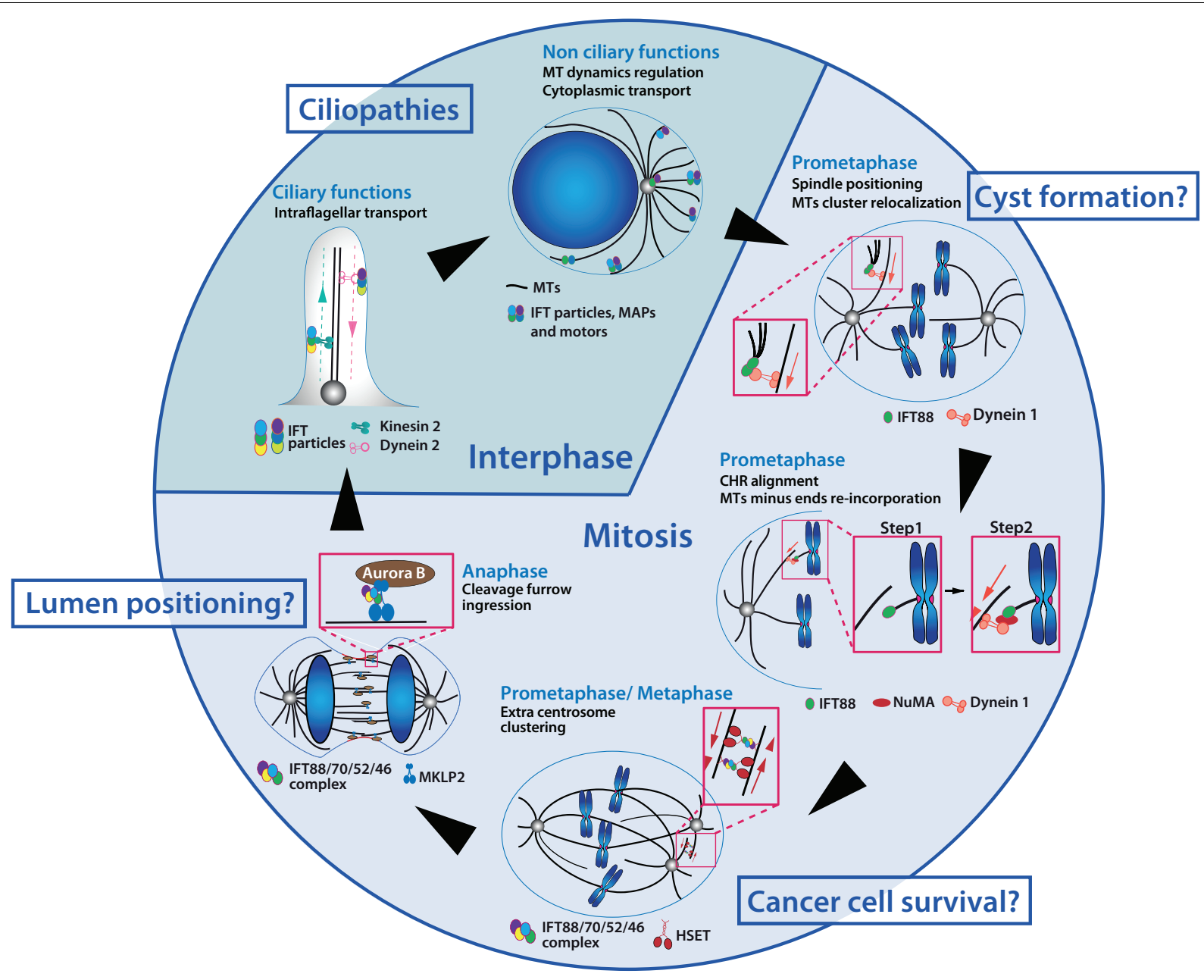

FIGURE 2 | IFT proteins function throughout the cell cycle in association with microtubules and motors. IFT proteins contribute to a variety of interphase and mitotic molecular mechanisms. IFT proteins are required for ciliogenesis and for the regulation of cytoplasmic microtubules which were both linked to ciliopathies related phenotypes. Novel non-ciliary mechanisms for IFT proteins in mitosis have been identified. Perturbation of those mechanisms may also be linked to developmental defects and diseases. MTs, microtubules; CHR, chromosome.

suggesting that other non-ciliary mechanisms could be involved. Indeed, depletion of the IFT-A protein IFT140 in mice leads to kidney cyst formation but without evidence of mitotic spindle orientation defects (Jonassen et al., 2012). Here, it should be noted that developmental defects related to IFT-A protein depletion may also be due to Wnt signaling defects that are independent of the cilia as shown in non-ciliated drosophila epithelial cells (Balmer et al., 2015; Vuong et al., 2018).

A recent work also strengthened the link between cell division defects and cyst formation. Indeed, Taulet et al. (2017) who characterized the role of IFT88 in cytokinetic cleavage furrow ingression and also showed that perturbations of cytokinesis geometry lead to lumen mispositioning and subsequently to multifocal lumen formation in 3D culture of LLC-PK1 kidney cells. These defects are reminiscent of the multifocal lumen defects observed in vivo, in the early stages of polycystic kidney disease, in a mouse model carrying IFT88 mutation (Moyer et al., 1994).
Altogether these works from animal models indicate that the cell division defects induced by IFT proteins perturbations may contribute to cystic kidney diseases. At this stage, additional studies will be required to assess the non-ciliary contribution of IFT proteins to other developmental defects associated with ciliopathies. Interestingly, Peralta et al. (2020) recently demonstrated that IFT88 and IFT20 are involved in embryonic cardiogenesis in both mice and zebrafish. This developmental defect is due to a non-ciliary role of IFT proteins involving the Hippo pathway effector Yap1 outside the cilium, within the cytoplasm.

The contribution of cytoplasmic non-ciliary processes to disease also emerged from the study of human pathologies. Indeed, IFT54 mutations that are responsible for nephronophthisis, a progressive fibrosis of the kidney tubules ending with terminal renal failure, were shown to result in excessive MAP4 expression and microtubule stabilization in patient fibroblasts, while having limited effects on cilia 
(Bizet et al., 2015). In addition, introduction of those mutations in mouse kidney IMCD-3 3D cellular model results in epithelialization and polarity defects, phenotypes frequently observed in kidney diseases (Schlüter and Margolis, 2012). Similarly, increased $\alpha$-tubulin acetylation, a posttranslational modification that is associated with stable and long lived microtubules (Piperno et al., 1987; Janke and Montagnac, 2017) was also found in human ARPKD cystic kidney samples and in cultured cells depleted of IFT88 (Berbari et al., 2013). While in this example ciliary defects occur concomitantly to the cytoplasmic microtubule phenotypes, it is worth noting that IFT proteins perturbation also affects cytoplasmic microtubules.

Finally, a recent study identified two mutations in IFT52 that cause short rib thoracic dysplasia and congenital anomaly of kidney and urinary tract. The authors showed, using mouse IMCD-3 cell culture, that these mutations perturb the interaction between IFT52 and centrin and trigger centriole splitting (Dupont et al., 2019). Additionally, they showed that IFT52 null IMCD-3 cells display defective cytoplasmic microtubule anchorage and dynamics. Of note, contrasting results were recently observed in human RPE-1 cells, where IFT52 depletion did not trigger centriole splitting in mitotic cells and did not affect microtubule dynamics in interphase cells (Vitre et al., 2020) indicating that the effects of IFT52 on those parameters could be cell type dependent.

Altogether, these works indicate that IFT proteins have microtubule-dependent functions outside the ciliary compartment. They also support the idea that non-ciliary functions may contribute, at least in part, to some developmental defects initially linked exclusively to cilia dysfunctions.

\section{REFERENCES}

Baker, S. A., Freeman, K., Luby-Phelps, K., Pazour, G. J., and Besharse, J. C. (2003). IFT20 links kinesin II with a mammalian intraflagellar transport complex that is conserved in motile flagella and sensory Cilia. J. Biol. Chem. 278, 34211-34218. doi: 10.1074/jbc.m300156200

Balmer, S., Dussert, A., Collu, G. M., Benitez, E., Iomini, C., and Mlodzik, M. (2015). Components of intraflagellar transport complex a function independently of the cilium to regulate canonical wnt signaling in Drosophila. Dev. Cell 34, 705-718. doi: 10.1016/j.devcel.2015.07.016

Barnes, B. G. (1961). Ciliated secretory cells in the pars distalis of the mouse hypophysis. J. Ultrasructure Res. 5, 453-467. doi: 10.1016/S0022-5320(61) 80019-1

Behal, R. H., Miller, M. S., Qin, H., Lucker, B. F., Jones, A., and Cole, D. G. (2012). Subunit interactions and organization of the Chlamydomonas reinhardtii intraflagellar transport complex A proteins. J. Biol. Chem. 287, 11689-11703. doi: 10.1074/jbc.M111.287102

Ben-Omran, T., Alsulaiman, R., Kamel, H., Shaheen, R., and Alkuraya, F. S. (2015). Intrafamilial clinical heterogeneity of CSPP1-related ciliopathy. Am. J. Med. Genet. Part A 167, 2478-2480. doi: 10.1002/ajmg.a. 37175

Berbari, N. F., Sharma, N., Malarkey, E. B., Pieczynski, J. N., Boddu, R., Gaertig, J., et al. (2013). Microtubule modifications and stability are altered by cilia perturbation and in cystic kidney disease. Cytoskeleton 70, 24-31. doi: 10.1002/ $\mathrm{cm} .21088$

Bhogaraju, S., Cajanek, L., Fort, C., Blisnick, T., Weber, K., Taschner, M., et al. (2013). Molecular basis of tubulin transport within the cilium by IFT74 and IFT81. Science 341, 1009-1012. doi: 10.1126/science.124 0985

\section{CONCLUDING REMARKS}

Overall, IFT proteins have to be considered as key regulators of ciliary and non-ciliary processes in association with microtubules and motors. Yet, we are only starting to understand the various roles these proteins may play beyond their role in cilia. Moreover, very little is known on the precise molecular mechanisms of these complexes as cargo and/or regulators of microtubules or ciliary and non-ciliary motors. Those regulatory functions of IFT proteins will have to be fully addressed, in the future, through in vitro reconstitution approaches. Finally, to further understand the exact contribution of non-ciliary functions of IFT proteins to ciliopathies it will now be essential to dynamically characterize the functional links between the non-ciliary functions of IFT proteins and 3D tissue architecture using 3D cell culture approaches and animal models.

\section{AUTHOR CONTRIBUTIONS}

$\mathrm{BV}, \mathrm{AG}$, and $\mathrm{BD}$ jointly wrote the manuscript. All authors contributed to the article and approved the submitted version.

\section{FUNDING}

This work was supported by ANR JCJC IRMM grant (ANR-18CE11-0025-01 to BV) and ANR PRC LUCELL grant (ANR-19CE13-0014-01 to BD).

Bhogaraju, S., Taschner, M., Morawetz, M., Basquin, C., and Lorentzen, E. (2011). Crystal structure of the intraflagellar transport complex 25/27. EMBO J. 30, 1907-1918. doi: 10.1038/emboj.2011.110

Bhogaraju, S., Weber, K., Engel, B. D., Lechtreck, K. F., and Lorentzen, E. (2014). Getting tubulin to the tip of the cilium: one IFT train, many different tubulin cargo-binding sites? BioEssays 36, 463-467. doi: 10.1002/bies.201400007

Bizet, A. A., Becker-Heck, A., Ryan, R., Weber, K., Filhol, E., Krug, P., et al. (2015). Mutations in TRAF3IP1/IFT54 reveal a new role for IFT proteins in microtubule stabilization. Nat. Commun. 6:8666.

Bloodgood, R. A. (2009). From Central to Rudimentary to Primary: The History of an Underappreciated Organelle Whose time has Come. The Primary Cilium, 1 Edn. Amsterdam: Elsevier, doi: 10.1016/s0091-679x(08)94001-2

Boehlke, C., Janusch, H., Hamann, C., Powelske, C., Mergen, M., Herbst, H., et al. (2015). A cilia independent role of Ift88/Polaris during cell migration. PLoS One 10:e0140378. doi: 10.1371/journal.pone.0140378

Boldt, K., Van Reeuwijk, J., Lu, Q., Koutroumpas, K., Nguyen, T. M. T., Texier, Y., et al. (2016). An organelle-specific protein landscape identifies novel diseases and molecular mechanisms. Nat. Commun. 7:11491. doi: 10.1038/ ncomms11491

Borovina, A., and Ciruna, B. (2013). IFT88 plays a cilia-and PCP-independent role in controlling oriented cell divisions during vertebrate embryonic development. Cell Rep. 5, 37-43. doi: 10.1016/j.celrep.2013.08.043

Buisson, J., Chenouard, N., Lagache, T., Blisnick, T., Olivo-Marin, J. C., and Bastin, P. (2013). Intraflagellar transport proteins cycle between the flagellum and its base. J. Cell Sci. 126, 327-338. doi: 10.1242/jcs.117069

Bujakowska, K. M., Zhang, Q., Siemiatkowska, A. M., Liu, Q., Place, E., Falk, M. J., et al. (2015). Mutations in IFT172 cause isolated retinal degeneration and Bardet-Biedl syndrome. Hum. Mol. Genet. 24, 230-242. doi: 10.1093/hmg/ ddu441 
Cole, D. G., Diener, D. R., Himelblau, A. L., Beech, P. L., Fuster, J. C., and Rosenbaum, J. L. (1998). Chlamydomonas kinesin-II-dependent intraflagellar transport (IFT): IFT particles contain proteins required for ciliary assembly in Caenorhabditis elegans sensory neurons. J. Cell Biol. 141, 993-1008. doi: 10.1083/jcb.141.4.993

Corbit, K. C., Aanstad, P., Singla, V., Norman, A. R., Stainier, D. Y. R., and Reiter, J. F. (2005). Vertebrate Smoothened functions at the primary cilium. Nature 437, 1018-1021. doi: 10.1038/nature04117

Craft, J. M., Harris, J. A., Hyman, S., Kner, P., and Lechtreck, K. F. (2015). Tubulin transport by IFT is upregulated during ciliary growth by a cilium-autonomous mechanism. J. Cell Biol. 208, 223-237. doi: 10.1083/jcb.201409036

Deane, J. A., Cole, D. G., Seeley, E. S., Diener, D. R., and Rosenbaum, J. L. (2001). Localization of intraflagellar transport protein IFT52 identifies basal body transitional fibers as the docking site for IFT particles. Curr. Biol. 11, 1586-1590. doi: 10.1016/s0960-9822(01)00484-5

Delaval, B., Bright, A., Lawson, N. D., and Doxsey, S. (2011). The cilia protein IFT88 is required for spindle orientation in mitosis. Nat. Cell Biol. 13, 461-468. doi: $10.1038 /$ ncb2202

Dupont, M. A., Humbert, C., Huber, C., Siour, Q., Guerrera, I. C., Jung, V., et al. (2019). Human IFT52 mutations uncover a novel role for the protein in microtubule dynamics and centrosome cohesion. Hum. Mol. Genet. 28, 2720-2737. doi: 10.1093/hmg/ddz091

Engel, B. D., Lechtreck, K. F., Sakai, T., Ikebe, M., Witman, G. B., and Marshall, W. F. (2009). Total Internal Reflection Fluorescence (TIRF) Microscopy of Chlamydomonas Flagella, 1 Edn. Amsterdam: Elsevier, doi: 10.1016/S0091679X(08)93009-0

Fawcett, D. W., and Porter, K. R. (1954). A study of the fine structure of ciliated epithelia. J. Morphol. 94, 221-281. doi: 10.1002/jmor.1050940202

Ferreira, R., Fukui, H., Chow, R., Vilfan, A., and Vermot, J. (2019). The cilium as a force sensor-myth versus reality. J. Cell Sci. 132:213496. doi: 10.1242/jcs.213496

Finetti, F., Paccani, S. R., Riparbelli, M. G., Giacomello, E., Perinetti, G., Pazour, G. J., et al. (2009). Intraflagellar transport is required for polarized recycling of the TCR/CD3 complex to the immune synapse. Nat. Cell Biol. 11, 1332-1339. doi: $10.1038 /$ ncb1977

Finetti, F., Patrussi, L., Masi, G., Onnis, A., Galgano, D., Lucherini, O. M., et al. (2014). Specific recycling receptors are targeted to the immune synapse by the intraflagellar transport system. J. Cell Sci. 127, 1924-1937. doi: 10.1242/jcs. 139337

Fischer, E., Legue, E., Doyen, A., Nato, F., Nicolas, J. F., Torres, V., et al. (2006). Defective planar cell polarity in polycystic kidney disease. Nat. Genet. 38, 21-23. doi: $10.1038 /$ ng 1701

Follit, J. A., Tuft, R. A., Fogarty, K. E., and Pazour, G. J. (2006). The intraflagellar transport protein IFT20 is associated with the Golgi complex and is required for cilia assembly. Mol. Biol. Cell 17, 3781-3792. doi: 10.1091/mbc.e06-02-0133

Funabashi, T., Katoh, Y., Michisaka, S., Terada, M., Sugawa, M., and Nakayama, K. (2017). Ciliary entry of KIF17 is dependent on its binding to the IFT-B complex via IFT46-IFT56 as well as on its nuclear localization signal. Mol. Biol. Cell 28, 624-633. doi: 10.1091/mbc.e16-09-0648

Funabashi, T., Katoh, Y., Okazaki, M., Sugawa, M., and Nakayama, K. (2018). Interaction of heterotrimeric kinesin-II with IFT-B-connecting tetramer is crucial for ciliogenesis. J. Cell Biol. 8:jcb.201801039-13.

Guo, C. W., Xiong, S., Liu, G., Wang, Y. F., He, Q. Y., Zhang, X. E., et al. (2010). Proteomic analysis reveals novel binding partners of MIPT3 in human cells. Proteomics 10, 2337-2347. doi: 10.1002/pmic.2010 00130

Halbritter, J., Bizet, A. A., Schmidts, M., Porath, J. D., Braun, D. A., Gee, H. Y., et al. (2013). Defects in the IFT-B component IFT172 cause jeune and mainzersaldino syndromes in humans. Am. J. Hum. Genet. 93, 915-925. doi: 10.1016/j. ajhg.2013.09.012

Hao, L., Thein, M., Brust-Mascher, I., Civelekoglu-Scholey, G., Lu, Y., Acar, S., et al. (2011). Intraflagellar transport delivers tubulin isotypes to sensory cilium middle and distal segments. Nat. Cell Biol. 13, 790-798. doi: 10.1038/nc b2268

Hirano, T., Katoh, Y., Nakayama, K., and Marshall, W. (2017). Intraflagellar transport-A complex mediates ciliary entry and retrograde trafficking of ciliary G protein-coupled receptors. Mol. Biol. Cell 28, 429-439. doi: 10.1091/mbc.E1611-0813
Hou, Y., Pazour, G. J., and Witman, G. B. (2004). A dynein light intermediate chain, DlbLIC, is required for retrograde intraflagellar transport. Mol. Biol. Cell 15, 4382-4394. doi: 10.1091/mbc.E04-05-0377

Howard, P. W., Jue, S. F., and Maurer, R. A. (2013). Interaction of mouse TTC30/DYF-1 with multiple intraflagellar transport complex B proteins and KIF17. Exp. Cell Res. 319, 2275-2281. doi: 10.1016/j.yexcr.2013. 06.010

Hua, K., and Ferland, R. J. (2018). Primary cilia proteins: ciliary and extraciliary sites and functions. Cell. Mol. Life Sci. 75, 1521-1540. doi: 10.1007/s00018-0172740-5

Huang, Q., Pu, M., Zhao, G., Dai, B., Bian, Z., Tang, H., et al. (2017). Tg737 regulates epithelial-mesenchymal transition and cancer stem cell properties via a negative feedback circuit between Snail and HNF4 $\alpha$ during liver stem cell malignant transformation. Cancer Lett. 402, 52-60. doi: 10.1016/j.canlet.2017. 05.005

Huang, Y., Zheng, J., Chen, D., Li, F., Wu, W., Huang, X., et al. (2017). Transcriptome profiling identifies a recurrent CRYL1-IFT88 chimeric transcript in hepatocellular carcinoma. Oncotarget 8, 40693-40704. doi: 10.18632/oncotarget.17244

Huangfu, D., Liu, A., Rakeman, A. S., and Murcia, N. S. (2003). Hedgehog signalling in the mouse requires intraflagellar transport proteins. Nature 426 , 83-87. doi: 10.1038/nature02080.1

Hueschen, C. L., Kenny, S. J., Xu, K., and Dumont, S. (2017). NuMA recruits dynein activity to microtubule minus-ends at mitosis. Elife 6, 1-26. doi: 10.7554/eLife. 29328

Imanishi, M., Endres, N. F., Gennerich, A., and Vale, R. D. (2006). Autoinhibition regulates the motility of the C. elegansintraflagellar transport motor OSM-3. J. Cell Biol. 174, 931-937. doi: 10.1083/jcb.200605179

Iomini, C., Babaev-Khaimov, V., Sassaroli, M., and Piperno, G. (2001). Protein particles in Chlamydomonas flagella undergo a transport cycle consisting of four phases. J. Cell Biol. 153, 13-24. doi: 10.1083/jcb.153.1.13

Iomini, C., Tejada, K., Mo, W., Vaananen, H., and Piperno, G. (2004). Primary cilia of human endothelial cells disassemble under laminar shear stress. J. Cell Biol. 164, 811-817. doi: 10.1083/jcb.200312133

Isfort, R. J., Cody, D. B., Doersen, C. J., Richards, W. G., Yoder, B. K., Wilkinson, J. E., et al. (1997). The tetratricopeptide repeat containing Tg737 gene is a liver neoplasia tumor suppressor gene. Oncogene 15, 1797-1803. doi: 10.1038/sj.onc. 1201535

Janke, C., and Montagnac, G. (2017). Causes and consequences of microtubule acetylation. Curr. Biol. 27, R1287-R1292. doi: 10.1016/j.cub.2017.10.044

Jékely, G., and Arendt, D. (2006). Evolution of intraflagellar transport from coated vesicles and autogenous origin of the eukaryotic cilium. BioEssays 28, 191-198. doi: 10.1002/bies.20369

Jonassen, J. A., San Agustin, J., Follit, J. A., and Pazour, G. J. (2008). Deletion of IFT20 in the mouse kidney causes misorientation of the mitotic spindle and cystic kidney disease. J. Cell Biol. 183, 377-384. doi: 10.1083/jcb.200808137

Jonassen, J. A., SanAgustin, J., Baker, S. P., and Pazour, G. J. (2012). Disruption of IFT Complex a causes cystic kidneys without mitotic spindle misorientation. J. Am. Soc. Nephrol. 23, 641-651. doi: 10.1681/ASN.2011080829

Katoh, Y., Terada, M., Nishijima, Y., Takei, R., Nozaki, S., Hamada, H., et al. (2016). Overall architecture of the intraflagellar transport (IFT)-B complex containing cluap1/IFT38 as an essential component of the IFT-B peripheral subcomplex. J. Biol. Chem. 291, 10962-10975. doi: 10.1074/jbc.M116.713883

Kishimoto, N., Cao, Y., Park, A., and Sun, Z. (2008). Cystic kidney gene seahorse regulates cilia-mediated processes and wnt pathways. Dev. Cell 14, 954-961. doi: 10.1016/j.devcel.2008.03.010

Kozminski, K. G., Beech, P. L., and Rosenbaum, J. L. (1995). The Chlamydomonas kinesin-like protein FLA10 is involved in motility associated with the flagellar membrane. J. Cell Biol. 131, 1517-1527. doi: 10.1083/jcb.131.6.1517

Kozminski, K. G., Johnson, K. A., Forscher, P., and Rosenbaum, J. L. (1993). A motility in the eukaryotic flagellum unrelated to flagellar beating. Proc. Natl. Acad. Sci. U.S.A. 90, 5519-5523. doi: 10.1073/pnas.90.12. 5519

Krock, B. L., and Perkins, B. D. (2008). The intraflagellar transport protein IFT57 is required for cilia maintenance and regulates IFT-particle-kinesinII dissociation in vertebrate photoreceptors. J. Cell Sci. 121, 1907-1915. doi: $10.1242 /$ jcs. 029397 
Kubo, T., Brown, J. M., Bellve, K., Craige, B., Craft, J. M., Fogarty, K., et al. (2016). Together, the IFT81 and IFT74 N-termini form the main module for intraflagellar transport of tubulin. J. Cell Sci. 129, 2106-2119. doi: 10.1242/jcs. 187120

Ledbetter, M. C., and Porter, K. R. (1963). A “microtubule" in plant cell fine structure. J. Cell Biol. 19, 239-250. doi: 10.1083/jcb.19.1.239

Ling, L., and Goeddel, D. V. (2000). MIP-T3, a Novel Protein Linking tumor necrosis factor receptor-associated factor 3 to the microtubule network. J. Biol. Chem. 275, 23852-23860. doi: 10.1074/jbc.m001095200

Liu, H., Kiseleva, A. A., and Golemis, E. A. (2018). Ciliary signalling in cancer. Nat. Rev. Cancer 18, 511-524. doi: 10.1038/s41568-0180023-6

Lucker, B. F., Behal, R. H., Qin, H., Siron, L. C., Taggart, W. D., Rosenbaum, J. L., et al. (2005). Characterization of the intraflagellar transport complex B core: direct interaction of the IFT81 and IFT74/72 subunits. J. Biol. Chem. 280, 27688-27696. doi: 10.1074/jbc.M505062200

Lucker, B. F., Miller, M. S., Dziedzic, S. A., Blackmarr, P. T., and Cole, D. G. (2010). Direct interactions of intraflagellar transport complex B proteins IFT88, IFT52, and IFT46. J. Biol. Chem. 285, 21508-21518. doi: 10.1074/jbc.M110.106997

Manton, I., and Clarke, B. (1952). An electron microscope study of the spermatozoid of sphagnum. J. Exp. Bot. 3, 265-275. doi: 10.1093/jxb/3.3.265

McFie, M., Koneva, L., Collins, I., Coveney, C. R., Clube, A. M., Chanalaris, A., et al. (2020). Ciliary proteins specify the cell inflammatory response by tuning NFкB signaling, independently of primary cilia. J. Cell Sci. 20:jcs.239871. doi: $10.1242 /$ jcs. 239871

McGrath, J., Somlo, S., Makova, S., Tian, X., and Brueckner, M. (2003). Two populations of node monocilia initiate left-right asymmetry in the mouse. Cell 114, 61-73. doi: 10.1016/S0092-8674(03)00511-7

Mohamed, M. A. A., Stepp, W. L., and Ökten, Z. (2018). Reconstitution reveals motor activation for intraflagellar transport. Nature 557, 387-391. doi: 10.1038/ s41586-018-0105-3

Moyer, J. H., Lee-Tischler, M. J., Kwon, H. Y., Schrick, J. J., Avner, E. D., Sweeney, W. E., et al. (1994). Candidate gene associated with a mutation causing recessive polycystic kidney disease in mice. Science 264, 1329-1333. doi: 10.1126/science. 8191288

Mukhopadhyay, S., Wen, X., Chih, B., Nelson, C. D., Lane, W. S., Scales, S. J., et al. (2010). TULP3 bridges the IFT-A complex and membrane phosphoinositides to promote trafficking of $\mathrm{G}$ protein-coupled receptors into primary cilia. Genes Dev. 24, 2180-2193. doi: 10.1101/gad.1966210

Pathak, N., Obara, T., Mangos, S., Liu, Y., and Drummond, I. A. (2007). The zebrafish fleer gene encodes an essential regulator of cilia tubulin polyglutamylation. Mol. Biol. Cell 18, 4353-4364. doi: 10.1091/mbc.e07-060537

Pazour, G. J., Dickert, B. L., Vucica, Y., Seeley, E. S., Rosenbaum, J. L., Witman, G. B., et al. (2000). Chlamydomonas IFT88 and its mouse homologue, polycystic kidney disease gene $\mathrm{Tg737}$, are required for assembly of cilia and flagella. J. Cell Biol. 151, 709-718. doi: 10.1083/jcb.151. 3.709

Pazour, G. J., Dickert, B. L., and Witman, G. B. (1999). The DHC1b (DHC2) isoform of cytoplasmic dynein is required for flagellar assembly. J. Cell Biol. 144, 473-481. doi: 10.1083/jcb.144.3.473

Pazour, G. J., Wilkerson, C. G., and Witman, G. B. (1998). A dynein light chain is essential for the retrograde particle movement of intraflagellar transport (IFT). J. Cell Biol. 141, 979-992. doi: 10.1083/jcb.141. 4.979

Pedersen, L. B., Geimer, S., Sloboda, R. D., and Rosenbaum, J. L. (2003). The microtubule plus end-tracking protein EB1 is localized to the flagellar tip and basal bodies in Chlamydomonas reinhardtii. Curr. Biol. 13, 1969-1974. doi: 10.1016/j.cub.2003.10.058

Pedersen, L. B., Miller, M. S., Geimer, S., Leitch, J. M., Rosenbaum, J. L., and Cole, D. G. (2005). Chlamydomonas IFT172 is encoded by FLA11, interacts with CrEB1, and regulates IFT at the flagellar tip. Curr. Biol. 15, 262-266. doi: $10.1016 /$ j.cub.2005.01.037

Peralta, M., Ortiz Lopez, L., Jerabkova, K., Lucchesi, T., Vitre, B., Han, D., et al. (2020). Intraflagellar transport complex b proteins regulate the hippo effector yap1 during cardiogenesis. Cell Rep. 32:107932. doi: 10.1016/j.celrep.2020. 107932
Perrone, C. A., Tritschler, D., Taulman, P., Bower, R., Yoder, B. K., and Porter, M. E. (2003). A novel dynein light intermediate chain colocalizes with the retrograde motor for intraflagellar transport at sites of axoneme assembly in chlamydomonas and mammalian cells. Mol. Biol. Cell 14, 2041-2056. doi: 10. 1091/mbc.e02-10-0682

Pigino, G., Geimer, S., Lanzavecchia, S., Paccagnini, E., Cantele, F., Diener, D. R., et al. (2009). Electron-tomographic analysis of intraflagellar transport particle trains in situ. J. Cell Biol. 187, 135-148. doi: 10.1083/jcb.20090 5103

Piperno, G., LeDizet, M., and Chang, X. J. (1987). Microtubules containing acetylated alpha-tubulin in mammalian cells in culture. J. Cell Biol. 104, 289302. doi: $10.1083 /$ jcb.104.2.289

Piperno, G., Siuda, E., Henderson, S., Segil, M., Vaananen, H., and Sassaroli, M. (1998). Distinct mutants of retrograde intraflagellar transport (IFT) share similar morphological and molecular defects. J. Cell Biol. 143, 1591-1601. doi: 10.1083/jcb.143.6.1591

Praetorius, H. A., and Spring, K. R. (2001). Bending the MDCK cell primary cilium increases intracellular calcium. J. Membr. Biol. 184, 71-79. doi: 10.1007/s00232001-0075-4

Praetorius, H. A., and Spring, K. R. (2003). Removal of the MDCK cell primary cilium abolishes flow sensing. J. Membr. Biol. 191, 69-76. doi: 10.1007/s00232002-1042-4

Prevo, B., Scholey, J. M., and Peterman, E. J. G. (2017). Intraflagellar transport: mechanisms of motor action, cooperation, and cargo delivery. FEBS J. 284, 2905-2931. doi: 10.1111/febs. 14068

Qin, H., Diener, D. R., Geimer, S., Cole, D. G., and Rosenbaum, J. L. (2004). Intraflagellar transport (IFT) cargo: IFT transports flagellar precursors to the tip and turnover products to the cell body. J. Cell Biol. 164, 255-266. doi: $10.1083 /$ jcb. 200308132

Ramsbottom, S. A., Miles, C., and Sayer, J. A. (2015). Murine Cep290 phenotypes are modified by genetic backgrounds and provide an impetus for investigating disease modifier alleles. F1000Research 4:590. doi: 10.12688/f1000research. 6959.1

Ramsbottom, S. A., Thelwall, P. E., Wood, K. M., Clowry, G. J., Devlin, L. A., and Silbermann, F. (2020). Mouse genetics reveals Barttin as a genetic modifier of Joubert syndrome. Proc. Natl. Acad. Sci. U.S.A. 117, 1113-1118. doi: 10.1073/ pnas. 1912602117

Reiter, J. F., and Leroux, M. R. (2017). Genes and molecular pathways underpinning ciliopathies. Nat. Rev. Mol. Cell Biol. 18, 533-547. doi: 10.1038/ nrm. 2017.60

Robert, A., Margall-Ducos, G., Guidotti, J. E., Bregerie, O., Celati, C., Brechot, C., et al. (2007). The intraflagellar transport component IFT88/polaris is a centrosomal protein regulating G1-S transition in non-ciliated cells. J. Cell Sci. 120, 628-637. doi: 10.1242/jcs.03366

Schlüter, M. A., and Margolis, B. (2012). Apicobasal polarity in the kidney. Exp. Cell Res. 318, 1033-1039. doi: 10.1016/j.yexcr.2012.02.028

Schou, K. B., Andersen, J. S., and Pedersen, L. B. (2014). A divergent calponin homology $(\mathrm{NN}-\mathrm{CH})$ domain defines a novel family: implications for evolution of ciliary IFT complex B proteins. Bioinformatics 30, 899-902. doi: 10.1093/ bioinformatics/btt661

Slautterback, D. B. (1963). Cytoplasmic microtubules. I. Hydra. J. Cell Biol. 18, 367-388. doi: $10.1083 /$ jcb.18.2.367

Sorokin, S. (1962). Centrioles and the formation of rudimentary cilia by fibroblasts and smooth muscle cells. J. Cell Biol. 15, 363-377. doi: 10.1083/jcb.15.2.363

Stepanek, L., and Pigino, G. (2016). Microtubule doublets are double-track railways for intraflagellar transport trains. Science 352, 721-724. doi: 10.1126/science. aaf4594

Taschner, M., Bhogaraju, S., and Lorentzen, E. (2012). Architecture and function of IFT complex proteins in ciliogenesis. Differentiation 83, S12-S22.

Taschner, M., Bhogaraju, S., Vetter, M., Morawetz, M., and Lorentzen, E. (2011). Biochemical mapping of interactions within the intraflagellar transport (IFT) B core complex: IFT52 binds directly to four other IFT-B subunits. J. Biol. Chem. 286, 26344-26352. doi: 10.1074/jbc.M111.254920

Taschner, M., Kotsis, F., Braeuer, P., Kuehn, E. W., and Lorentzen, E. (2014). Crystal structures of IFT70/52 and IFT52/46 provide insight into intraflagellar transport B core complex assembly. J. Cell Biol. 207, 269-282. doi: 10.1083/jcb. 201408002 
Taschner, M., Lorentzen, A., Mourão, A., Collins, T., Freke, G. M., Moulding, D., et al. (2018). Crystal structure of intraflagellar transport protein 80 reveals a homodimer required for ciliogenesis. Elife 7, 1-27. doi: 10.7554/eLife. 33067

Taschner, M., and Lorentzen, E. (2016). The intraflagellar transport machinery. Cold Spring Harb. Perspect. Biol. 8:a028092-20.

Taschner, M., Weber, K., Mourao, A., Vetter, M., Awasthi, M., Stiegler, M., et al. (2016). Intraflagellar transport proteins $172,80,57,54,38$, and 20 form a stable tubulin-binding IFT-B2 complex. EMBO J. 35, 773-790. doi: 10.15252/embj. 201593164

Taulet, N., Douanier, A., Vitre, B., Anguille, C., Maurin, J., Dromard, Y., et al. (2019). IFT88 controls NuMA enrichment at k-fibers minus-ends to facilitate their re-anchoring into mitotic spindles. Sci. Rep. 9:46605. doi: 10.1038/s41598019-46605-x

Taulet, N., Vitre, B., Anguille, C., Douanier, A., Rocancourt, M., Taschner, M., et al. (2017). IFT proteins spatially control the geometry of cleavage furrow ingression and lumen positioning. Nat. Commun. 8:1479. doi: 10.1038/s41467017-01479-3

van Dam, T. J. P., Townsend, M. J., Turk, M., Schlessinger, A., Sali, A., Field, M. C., et al. (2013). Evolution of modular intraflagellar transport from a coatomerlike progenitor. Proc. Natl. Acad. Sci. U.S.A. 110, 6943-6948. doi: 10.1073/pnas. 1221011110

Varjosalo, M., and Taipale, J. (2008). Hedgehog: functions and mechanisms. Genes Dev. 22, 2454-2472. doi: 10.1101/gad.1693608

Vertii, A., Bright, A., Delaval, B., Hehnly, H., and Doxsey, S. (2015). New frontiers: discovering cilia-independent functions of cilia proteins. EMBO Rep. 16, 12751287. doi: 10.15252 /embr.201540632

Vitre, B., Taulet, N., Guesdon, A., Douanier, A., Dosdane, A., Cisneros, M., et al. (2020). IFT proteins interact with HSET to promote supernumerary centrosome clustering in mitosis. EMBO Rep. 21:e49234. doi: 10.15252/embr. 201949234

Vuong, L. T., Iomini, C., Balmer, S., Esposito, D., Aaronson, S. A., and Mlodzik, M. (2018). Kinesin-2 and IFT-A act as a complex promoting nuclear localization of $\beta$-catenin during Wnt signalling. Nat. Commun. 9:7605. doi: 10.1038/s41467018-07605-z

Wachter, S., Jung, J., Shafiq, S., Basquin, J., Fort, C., Bastin, P., et al. (2019). Binding of IFT22 to the intraflagellar transport complex is essential for flagellum assembly. EMBO J. 38, 1-18. doi: 10.15252/embj.201810 1251

Walther, Z., Vashishtha, M., and Hall, J. L. (1994). The Chlamydomonas FLA10 gene encodes a novel kinesin-homologous protein. J. Cell Biol. 126, 175-188. doi: $10.1083 /$ jcb.126.1.175

Wang, Z., Fan, Z.-C., Williamson, S. M., and Qin, H. (2009). Intraflagellar transport (IFT) protein IFT25 is a phosphoprotein component of IFT complex B and physically interacts with IFT27 in Chlamydomonas. PLoS One 4:e5384. doi: 10.1371/journal.pone.0005384

Weghe, J. C., Van De Harris, J. A., Kubo, T., Witman, G. B., and Lechtreck, K. F. (2020). Diffusion rather than IFT likely provides most of the tubulin required for axonemal assembly. J Cell Sci. 14:jcs.249805. doi: 10.1242/jcs.249805

Wood, C. R., Wang, Z., Diener, D., Zones, J. M., Rosenbaum, J., and Umen, J. G. (2012). IFT proteins accumulate during cell division and localize to the cleavage furrow in chlamydomonas. PLoS One 7:30729. doi: 10.1371/journal. pone.0030729

Yoshiba, S., Shiratori, H., Kuo, I. Y., Kawasumi, A., Shinohara, K., Nonaka, S., et al. (2012). Cilia at the node of mouse embryos sense fluid flow for left-right determination via $\mathrm{Pkd}$ 2. Science 338, 226-231. doi: 10.1126/science.1222538

You, N., Tan, Y., Zhou, L., Huang, X., Wang, W., Wang, L., et al. (2017). Tg737 acts as a key driver of invasion and migration in liver cancer stem cells and correlates with poor prognosis in patients with hepatocellular carcinoma. Exp. Cell Res. 358, 217-226. doi: 10.1016/j.yexcr.2017.06.021

Zhu, X., Liang, Y., Gao, F., and Pan, J. (2017). IFT54 regulates IFT20 stability but is not essential for tubulin transport during ciliogenesis. Cell. Mol. Life Sci. 17, 1-13. doi: 10.1016/0012-1606(75)90082-2

Conflict of Interest: The authors declare that the research was conducted in the absence of any commercial or financial relationships that could be construed as a potential conflict of interest.

Copyright (c) 2020 Vitre, Guesdon and Delaval. This is an open-access article distributed under the terms of the Creative Commons Attribution License (CC BY). The use, distribution or reproduction in other forums is permitted, provided the original author(s) and the copyright owner(s) are credited and that the original publication in this journal is cited, in accordance with accepted academic practice. No use, distribution or reproduction is permitted which does not comply with these terms. 